\title{
Neck Muscle Vibration Alters Visually Perceived Roll in Normals
}

\author{
George J. McKenna, ${ }^{1}$ Grace C. Y. Peng, ${ }^{2}$ and David S. Zee ${ }^{2,3}$ \\ ${ }^{1}$ Department of Neurology, National Naval Medical Center, Bethesda, MD 20889, USA \\ ${ }^{2}$ Department of Neurology, The Johns Hopkins Hospital, Baltimore, MD 21287, USA \\ ${ }^{3}$ Department of Otolaryngology-Head and Neck Surgery, The Johns Hopkins Hospital, Baltimore, MD 21287, USA
}

Received: 19 February 2003; Accepted: 24 July 2003; Online publication: 23 October 2003

\section{ABSTRACT}

The objective of this study was to determine whether vibration of dorsal neck muscles or of the mastoid bone or of both modified the perception of visual orientation in the head roll-tilt plane in normal subjects. Measurements of the subjective visual vertical (SVV) were obtained from 26 normal human subjects. Subjects reported the SVV in the upright and in the left and right $30^{\circ}$ static head roll-tilt positions. Subjects then reported the SVV while vibration was applied to the left or right dorsal neck or left or right mastoid. Both head position and vibration independently modified settings of the SVV. In head-tilted positions, vibration of the upper dorsal neck muscles (on the side of the head opposite to the head tilt) caused a significantly greater shift of the SVV in the opposite direction of head roll-tilt compared to vibration of the lower dorsal neck muscles or of the mastoid. These results support a role for cervical somatosensory information in perception of visual orientation in the roll plane. Our findings may help explain the differences observed in visual orientation perception in normal subjects between head alone and whole-body roll-tilt. Finally, vibration of neck muscles in the head roll-tilted plane may be a useful method to test cervical somatosensory function possibly by increasing their response to external stimulation.

The views expressed in this article are those of the author and do not necessarily reflect the official policy or position of the Department of the Navy, Department of Defense, nor the U. S. Government.

Correspondence to: Dr. George J. McKenna • Neurology Service • National Naval Medical Center $\bullet 8901$ Wisconsin Avenue • Bethesda, MD 20889. email: gjmckenna@bethesda.med.navy.mil
Keywords: subjective visual vertical, vibration, mastoid, otolith, proprioception, neck roll-tilt

\section{INTRODUCTION}

Normal subjects in the upright position can align the subjective visual vertical (SVV) within $2^{\circ}-3^{\circ}$ of gravitational (earth) vertical (Friedmann 1970; Dai et al. 1989; Tribukait et al. 1996; Karlberg et al. 2002). When rotated to a tilted position in the roll plane, normal subjects become less accurate in aligning the SVV to gravitational vertical. Typically, at small angles of roll-tilt $\left(<60^{\circ}\right)$ there is a Müller or E-effect (aligning the SVV in the opposite direction of tilt) and at $>60^{\circ}$ of roll-tilt, there is an Aubert or A-effect (aligning the SVV in the direction of tilt) (Aubert 1861; Müller 1916; Witkin and Asch 1948; de Graff et al. 1992; Betts and Curthoys 1998). The reasons for these deviations are not fully understood but a diminished otolith sensitivity (the primary vestibular input in static roll-tilt) in roll-tilt likely plays a role (Markham 1989). Other sensory inputs besides vestibular must be involved since the SVV is influenced not only by the degree of rotation in the roll plane but by whether the head and body are rotated en bloc, or just the head, with the body remaining upright. For example, normal subjects align the subjective visual further in the opposite direction of roll-tilt (in the direction of an E-effect) when their head alone is rolltilted versus whole-body roll-tilt of the same magnitude (Wetzig and von Baumgarten 1990; Wade 1968; Guerrez et al. 1998a). These results suggest that somatosensory inputs from the neck contribute to the 
perception of upright (Wade 1968; Wetzig and von Baumgarten 1990).

Vibration of either the skull (such as the mastoid) or the neck [the sternocleidomastoid (SCM) or dorsal neck muscles typically] can alter visual orientation perception in normal subjects. The location seems important as vibration of different neck and head regions give different visual illusions (Lackner and Graybiel 1974; Taylor and McCloskey 1991; Biguer et al. 1998). Precisely which sensory system(s) are stimulated by vibration is unclear because vibration can stimulate both vestibular and muscle spindle afferents. Vibration can stimulate vestibular afferents such as triggering the vestibulocollic reflex. Halmagyi et al. (1995) induced short-latency myogenic potentials in the SCM muscle in humans by tapping the forehead. These vestibulocollic reflex potentials are absent when the vestibular nerve is sectioned, and they likely originate in a bone-conducted vibration wave stimulating the saccule. Vibration can also stimulate muscle spindle afferents causing healthy subjects to erroneously perceive that the vibrated muscle has lengthened and their limb has moved (Goodwin et al. 1972; Roll and Vedel 1982). This effect is caused by muscle vibration triggering activity in primary muscle spindle afferents which is sensed as further stretching of the muscle (Bianconi and van der Meulen 1963; Burke et al. 1976; Roll and Vedel 1982). Biguer et al. (1998) demontrated that dorsal neck vibration in the upright position causes a visual illusion of displacement of a visual target away from the side of vibration in normal subjects and suggested this was due to neck muscle proprioceptive stimulation. This illusory contralateral displacement of a visual target with neck vibration is magnified in vestibular-deficient patients compared with normals (Strupp et al. 1998; Karlberg et al. 2002). This magnified cervical afferent effect may be related to a compensatory response for a lack of labyrinthine inputs (Bless and De Graaf 1991; Strupp et al. 1998).

Recently, Betts el al. (2000) studied SCM and mastoid vibration in the roll plane using whole body roll-tilt. They found vibration but not static wholebody roll-tilt modified visual orientation perception in unilateral vestibular-deficient (UVD) patients; while in normal subjects, static whole-body tilt but not vibration modified visual orientation perception. The authors concluded that SCM stimulation does not contribute to visual orientation perception in the roll plane in normal subjects. We wondered whether this lack of a neck vibration effect on visual perception in healthy subjects holds when only the head alone is roll-tilted. We hypothesized that if neck somatosensory afferents are important in the observed greater shift of visual perception in the direction opposite of roll-tilt when the head alone is roll-tilted versus whole- body roll-tilt, then we would likely see a vibrationinduced modification of visual perception under these testing conditions. We chose to stimulate dorsal neck muscles (instead of the SCM) because of evidence of their primary role in head-tilt in the roll plane (Mayoux-Benhamou et al. 1997). We included mastoid vibration so that a direct comparison to dorsal neck vibration could be made which may either further support or refute a primary cervical afferent effect in visual perception in the roll plane.

\section{METHODS}

\section{Subjects}

We studied 26 normal human subjects (ages 23-54, mean 36). There were 20 men and 6 women. Subjects had no history of dizziness and had normal vestibuloocular responses (VOR) to brief, high-acceleration head rotations (head thrust maneuver) (Halmagyi et al. 1990). Informed consent was obtained after a full explanation of the experimental procedure was given.

\section{Testing procedures}

All subjects were seated in a chair and their heads were stabilized in a given position using a bite bar made of dental impression material. The head was held either in the upright or in a $30^{\circ}$ left or a $30^{\circ}$ right head roll-tilt position. The trunk was always in the upright sitting position. In front of the subjects was a translucent rectangular screen, $120 \mathrm{~cm}$ wide and $110 \mathrm{~cm}$ high, on which a laser-generated line was rear-projected in an otherwise darkened room. The screen was $120 \mathrm{~cm}$ away from the subject. Two lasers were used. One projected a red line that was $2 \mathrm{~mm}$ wide and $88 \mathrm{~mm}$ long. A second laser of greater luminance projected a red dot $2 \mathrm{~mm}$ in diameter which was superimposed on the line along the center of the axis of rotation of the line. The red dot/line laser images were positioned on the screen to be level with the straight-ahead position for the subjects. Subjects were asked to maintain their line of sight where the dot and line laser were superimposed. The luminance of the projected red laser line was $1.00 \mathrm{~cd} / \mathrm{m}^{2}$, and of the red laser dot, $8.5 \mathrm{~cd} / \mathrm{m}^{2}$. A plumb line was used to ensure the laser line was aligned with earth vertical.

Subjects were asked to align the red laser line to earth vertical using a hand held computer mouse that controlled the orientation of the red laser line. For each testing angle, with or without vibration, subjects rotated the line to their earth-perceived vertical. When the line appeared earth vertical, they would click a button on the computer mouse, which would simultaneously record the measurement (accurate to 
within $0.1^{\circ}$ ). The red laser line was then offset, approximately $20^{\circ}-40^{\circ}$ clockwise or counterclockwise, in random order. After 15 settings of the SVV, the laser automatically shut off. The lights within the room were then turned on and either the position of the subject's head or the location of the vibrator or both were changed, and another set of 15 SVV settings was obtained. Initially the SVV was measured without vibration in each of the three head roll-tilt positions, upright and $30^{\circ}$ to the left or right, which were applied in random order. The head was positioned using an inclinometer placed atop the bite bar. Subjects then were tested in each of these three head positions with a handheld vibrator applied either to the right or left dorsal neck or to the right or left mastoid process, presented in random order. Hence, for each of the three head positions, data were collected first without vibration and then with left dorsal neck, left mastoid, right dorsal neck, and right mastoid vibration in random order. Thus, a total of 15 combinations (or trials) of the position of the head and location of vibration were tested and each combination included $15 \mathrm{SVV}$ setting measurements.

A commercially available battery-operated handheld vibrator (Brookstone's Mini Muscle Massager) was used. It had a frequency of vibration of $100 \mathrm{~Hz}$ and amplitude of approximately $1 \mathrm{~mm}$ (measured with a strobe light). The vibrator contacted a circular area on the skin measuring $3 \mathrm{~cm}$ in diameter. To ensure that battery life would not affect the effectiveness of the vibrator, the batteries were changed after every second subject. The vibrator was applied to the dorsal neck muscles approximately $2-5 \mathrm{~cm}$ below the occipital skull and $2-5 \mathrm{~cm}$ lateral to the cervical spine. When testing vibration of the dorsal neck, the vibrator was never in direct contact with the skull. The vibrator was placed directly behind the pinna of the ear to test mastoid vibration. The authors always assisted the subjects in placing the vibrator on the proper location to be tested prior to each trial. The authors then left the room prior to testing. Subjects applied the vibrator to each site and applied enough pressure to maintain the vibrator firmly against the mastoid or dorsal neck during each of the 15 trials. Prior to turning the lights off, nearly all subjects noted that vibration of the mastoid or dorsal neck caused an illusion of movement of the room. This illusion was variable in direction and whether objects appeared to rotate or translate.

Typically, each of the 15 SVV setting measurements in one combination of head roll-tilt angle and vibration locale took 1-2 minutes to complete. Before each trial run, there was about 2 minutes during which the room lights were turned on and the angle of the head or the location of the vibrator was adjusted. Turning the lights on also prevented dark adaptation. Subjects did not wear framed spectacles during testing because of possible optical distortion, though they did wear their contact lenses if they had them. In all subjects, the line and dot projected by the laser were easily seen without spectacles. Viewing was always binocular.

\section{Data analysis}

The SVV measurement used for each subject in each head position with or without vibration was calculated as the mean of the $15 \mathrm{SVV}$ settings. The effects of vibration were calculated as the difference in means between the measures of the SVV with and without vibration for a given head angle. This difference (or shift) in SVV settings caused by vibration was analyzed using a one-way analysis of variance (ANOVA). Positive values represent clockwise rotations of the SVV relative to the subjects' perception of the projected laser line in front of them. Counterclockwise values are represented by negative values.

\section{RESULTS}

All control subjects could align the laser-generated line within $\pm 2.3^{\circ}$ of earth vertical with the head in the upright position (mean $=-0.3^{\circ}, 1.2 \mathrm{SD}$ in degrees) . These measurements are similar to those of normal controls reported by others (see Introduction). ANOVA of normal subject data showed main effects of head roll-tilt angle $(F=10.42 ; d f=2,46 ; p<0.01)$ and vibration $(F=3.96 ; d f=3.69 ; p=0.01)$ and no interaction between head roll-tilt and vibration $(F=0.35 ; d f=6.138 ; p>0.05)$ (Fig. 1$)$.

In the head roll-tilted positions, subjects set the SVV within $1^{\circ}$ of earth vertical when vibration was not applied (left head-tilt mean $=-0.40^{\circ}, 2.81$ SD in degrees; right head-tilt mean $=-0.67^{\circ}, 3.24 \mathrm{SD}$ in degrees). When the head was roll-tilted $30^{\circ}$ and vibration was applied, there was a different pattern of response depending upon whether the head was tilted to the left or the right (Fig. 1). Vibration shifted the SVV in the opposite direction of head roll-tilt (in direction of an E-effect) regardless of the site of vibration, but the shift was greater with right than with left head roll-tilt (Fig. 1).

Vibration caused a main effect as well. ANOVA with Bonferroni's method showed that the main effect was in neck and not mastoid vibration $(t=-3.21$; difference of means $=-1.05 ; p<0.05)$. The results shown in Figure 1 suggested a possible explanation for this primary neck vibration effect. The largest vibration-induced shifts of the SVV corresponded to vibration of the dorsal neck opposite to the side of head roll-tilt. Hence, the largest shifts in SVV 


\section{Vibration Effects}
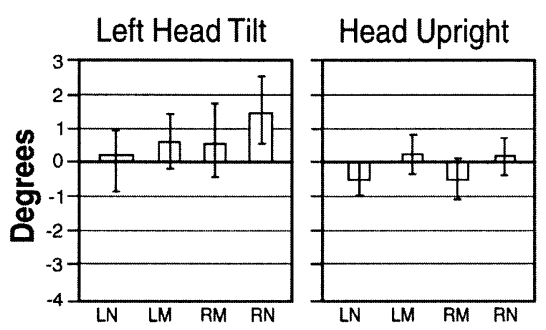

\section{Right Head Tilt}
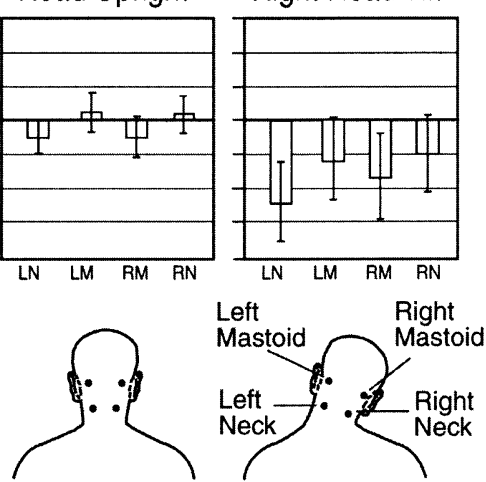

FIG. 1. Vibration effects in head roll-tilt in 26 normal controls. Vibration effects refers to the shift in the SVV induced by vibration of either the mastoid or the posterior neck when compared with nonvibration SVV measurements for each head roll-tilt position. Positive values (in degrees) represent shift of the SVV in the clockwise direction relative to the subject's viewpoint. Negative values are counterclockwise shift. Sites of vibration in each head roll-tilt position were as follows: $\mathrm{LN}=$ left neck, $\mathrm{LM}=$ left mastoid, $\mathrm{RM}=$ right mastoid, $\mathrm{RN}=$ right neck. Error bars refer to 95 confidence intervals.

occurred when the right dorsal neck was vibrated in left head roll-tilt and left dorsal neck vibrated in right head roll-tilt. We designated these vibration sites "upper." "Lower" referred to vibration on the same side as the direction of roll-tilt. To determine the significance of "upper" versus "lower" vibration effects, we compared these results to left versus right vibration effects. In each case, we combined left head roll-tilted data with right head roll-tilted data by inverting the sign of right head roll-tilt to give a pooled data in head roll-tilted positions. We then grouped data to upper versus lower (Fig. 2A) vibration sites or to left versus right vibration sites (Fig. 2B). ANOVA revealed a main effect of upper versus lower neck $(F=2.76 ; d f=3,25 ; p<0.05)$ (Fig. 2A). Similarly, ANOVA comparing combined right and left head roll-tilted data when right versus left neck vibration data were combined did not show a statistically significant effect $(F=0.14 ; d f=3,25 ; p>0.05)$ (Fig. 2B).

\section{DISCUSSION}

In this study we found that normal subjects shifted the SVV significantly more in the direction opposite of head roll-tilt (in the same direction of an E-effect) when vibration was applied to the upper dorsal neck than to the lower dorsal neck. This finding suggests that dorsal neck vibration modifies visual perception orientation in the roll plane. Like the study of Betts et al. (2000), we found that roll-tilt position influenced SVV measurements. However, our finding of

\section{Left and Right Head Tilt Combined}
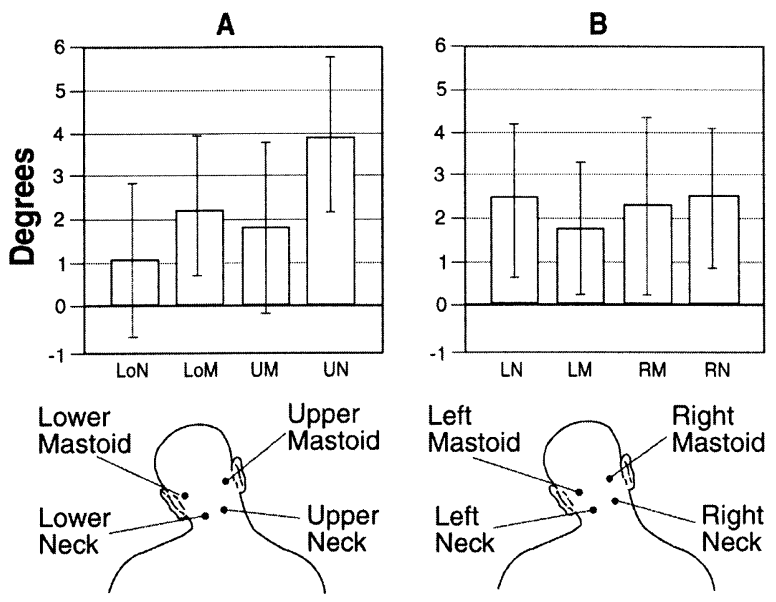

FIG. 2. Depiction of head $30^{\circ}$ static roll-tilted data combined in two different ways for the 26 normal controls. In both $\mathbf{A}$ and $\mathbf{B}$, all head roll-tilted data are transformed as if in left head roll-tilt. A. Head roll-tilted data was combined such that the location of vibration compared was "upper" versus "lower." B. Head roll-tilted data are combined such that the location of vibration compared was "left" versus "right." Sites of vibration in A were LoN = "lower" neck, LoM = "lower" mastoid, UM = "upper" mastoid, $\mathrm{UN}=$ "upper" neck. Sites of vibration in B were LN = left neck, $\mathrm{LM}=$ left mastoid, $\mathrm{RM}=$ right mastoid, $\mathrm{RN}=$ right neck. Error bars refer to 95 confidence intervals (see text for details).

an independent effect of vibration on SVV measurements in normal subjects differs from their results. There are several differences in the design between our two studies, including (1) the choice of neck muscle site vibrated (SCM versus dorsal neck muscles), (2) the visual perception measure employed [subjective visual horizontal (SVH) versus SVV], (3) vibratory apparatus used, and (4) whole-body roll-tilt versus head-alone roll-tilt. Although any or all of these differences in study design may have contributed to our different findings of neck vibration influences in visual perception in normal subjects, we believe the most likely possibility relates to wholebody versus head-only roll-tilt.

Recall that normal subjects align the SVV further in the opposite direction of roll-tilt when their head alone is tilted than when the whole body is roll-tilted the same amount (Wade 1968; Wetzig and von Baumgarten et al. 1990; Guerrez et al. 1998a). If cervical somatosensory afferents are partly responsible for this observation, then the visual illusion created by stimulation of neck muscle spindles via vibration may explain our findings where upper neck muscles significantly shifted the SVV in the E-effect direction. Goodwin et al. (1972) showed that vibration-induced illusions are best produced when the muscle is near its physiological maximum length. These upper neck muscles are likely nearer their maximal physiologic stretch and consequent sensi- 
tivity to vibration when in a head roll-tilt static position. Vibration of these stretched dorsal neck muscles in a head roll-tilted position might be expected to give an illusion of further stretching of the muscle and perception of further head roll-tilt. One might suspect that subjects would compensate for this illusion by adjusting the SVV in the opposite direction to the perceived change in head tilt, in effect shifting the SVV in the same direction as an E-effect. A similar finding may not be seen in the upright position because these muscles are not stretched near their physiologic maximum, hence, they are less sensitive to vibration. Likewise, in whole-body roll-tilt (as in Betts et al. 2000), neck muscles such as the SCM are not stretched and vibration may not be able to induce a significant effect on visual perception.

Betts et al. (2000) and our studies vibrated different neck muscles using different vibratory apparati and measures of visual orientation perception (SVH versus SVV). Both the SCM and the dorsal neck muscles (semispinalis capitus, transversospinalis, and splenius capitus) are involved in rotation of the head in the roll plane (Moore 1980; Mayoux-Benhamou et al. 1997). Both Betts et al. (2000) and we found that vibration of these muscles can modify visual orientation perception in the roll plane. However, we are unaware of a direct comparison of the effects of dorsal neck and SCM vibration in visual orientation perception in the same subjects. Most studies of visual perception utilizing vibration of neck muscles have measured changes in the yaw plane, not in the roll plane. Vibration of dorsal neck muscles (Biguer et al. 1988; Karnath et al. 1994; Strupp et al. 1998; Popov et al. 1999) and SCM muscles (Lackner and Levine 1979) appears to cause apparent motion of an illusory object away from the side of vibration suggesting that stimulation of these muscles modifies visual perception in a similar way. Of these studies, only Lackner and Levine (1979) also found a component of tilt in the roll plane, perhaps because they used an illuminated line versus an illuminated spot in these other studies. The proximity of the tendon insertions of the SCM and of the dorsal neck muscles also makes it difficult to determine which muscles (including possibly both SCM and dorsal neck together) were potentially stimulated via spread of a vibration wave (Biguer et al. 1988; Taylor and McCloskey 1991). At a $30^{\circ}$ roll-tilt angle, $\mathrm{SVH}$ gives slightly more of an Eeffect direction shift than SVV (Betts and Curthoys 1998). Could this greater baseline E-effect in SVH measurements without vibration obscure small changes induced by vibration in the E-effect direction? Finally, although the vibration devices used in the two studies had different amplitudes $(0.4 \mathrm{~mm}$ versus $1.0 \mathrm{~mm}$ ) and surface areas $(25 \mathrm{~mm}$ versus 30 $\mathrm{mm}$ in diameter), their frequencies were identical at
$100 \mathrm{~Hz}$. This frequency is sufficient to stimulate muscle spindles (Roll and Vedel 1982) and vestibular afferents (Young et al. 1977). Nearly all our subjects noted a visual illusion when vibration occurred with the lights on, suggesting that our vibration stimulus was adequate. The effects of the impact surface area and amplitude of vibration on our results are unclear. In conclusion, the discrepancies between our study and that of Betts et al. (2000) probably can be resolved only by comparing the same subjects in the same experimental setup with both the head tilted and whole body tilted.

An unexpected finding in our study was the asymmetric magnitude of SVV shift when vibration was applied in left versus right head roll-tilt. Although we can explain more easily the finding that vibration in static head-alone roll-tilt shifts the SVV in the Eeffect direction regardless of direction of tilt, the explanation for a greater magnitude of shift with right compared with left head roll-tilt is less obvious. This asymmetry has been noted in measures of ocular counter-roll (OCR) in normals where there was a greater OCR with left than with right roll-tilt of the same amount (Kompanejetz 1928; Diamond et al. 1979). Several studies have found a slight but significant counterclockwise deviation of the earth vertical when the head is in the upright position (Bauermeister 1964; Dichgans et al. 1974; Guerrez et al. 1998b). These findings and the fact that E- and A-effects in roll-tilt can be different from subject to subject have led to the suggestion of a "directional preponderence" in roll-tilt (Diamond et al. 1979; Betts and Curthoys 1998). This observation of asymmetry needs to be carefully considered when comparing normal subject data under different study designs in the roll plane (Wade 1969) and with other groups such as those with vestibular deficiencies.

We cannot exclude the possibility that our findings of a significant modification of visual perception in the head-static roll-tilt with upper neck vibration was not, in part, secondary to vestibular afferent stimulation. Karlberg et al. (2003), using scleral search coils in chronic unilateral vestibular deficit (UVD) subjects tested in the upright position in a lighted room, demonstrated that vibration produced similar ocular torsion magnitude and direction (with the upper pole of the eye rotating ipsilesionally) regardless of whether ipsilesional or contralesional vibration of the SCM or mastoid occurred. Furthermore, they found that in darkness vibration of the SCM or mastoid induced nystagmus which aligned with the plane of the intact semicircular canals (SCC) in two versus three SCC vestibular loss patients. Their results suggest that in chronic UVD patients, at least, vibration of neck muscles or mastoid stimulates vestibular afferents. What is unclear is why Karlberg et al. (2003) did not 
replicate the findings of their earlier work or that of others (Strupp et al. 1998; Betts et al. 2000; Karlberg et al. 2002) of a greater subjective visual shift with ipsilesional neck vibration by finding a greater ocular torsion under these circumstances. One explanation offered is that maximal ocular torsion takes longer than the $10 \mathrm{~s}$ used to measure ocular torsion in their study (typically 1-2 minutes is used in studies using subjective visual measurements) (Karlberg et al. 2003). Another possible explanation for the larger perceptual effects in the ipsilesional neck muscle vibrated in these other studies compared to the measured ocular torsion by Karlberg et al. (2003) represents an added neck afferent influence in visual perception. Karlberg et al. (2003) did not simultaneously measure the shift in SVH and ocular torsion which may have helped answer this question.

In conclusion, dorsal neck vibration modifies visual orientation perception in the roll plane. The finding that vibration of the upper neck shifted the SVV significantly more than lower neck in comparison with vibration of the mastoid suggests that somatosensory afferents from the neck were stimulated primarily by vibration. The use of head roll-tilt instead of whole-body roll-tilt may increase the sensitivity of vibration techniques by testing muscles in a stretched condition. Our findings support a role for cervical somatosensory afferents in roll-tilt and may help explain the differences observed in visual orientation perception when only the head versus the whole body is roll-tilted.

\section{ACKNOWLEDGMENTS}

This work was supported by NIH grant DC00979 (DSZ and GCYP). We thank Lloyd B. Minor, M.D., and Philip Cremer, M.D., for helpful discussion. We thank Adrian Lasker and Dale Roberts for technical support, Cara Olsen for statistical support, and Diana Pino for graphic design support.

\section{REFERENCES}

Aubert H. Eine scheinbare bedeutende Drehung von Objekten bei neigung des Kopfes nach rechts oder links. Virchows Arch. 20:381-393, 1861 .

BAUERMEISTER M. Effect of body tilt on apparent vertically, apparent body position and their relation. J. Exp. Psychol. 67:142-147, 1964.

BEtTs GA, Curthoys IS. Visually perceived vertical and visually perceived horizontal are not orthogonal. Vision Res. 38:19891999, 1998.

Betts Ga, Barone M, Karlberg M, MacDougall H, Curthoys IS. Neck muscle vibration alters visually-perceived roll after unilateral vestibular loss. Neuroreport 11(12):2659-2662, 2000.

Bianconi R, van Der Meulen JR. The response to vibration of the end organ on mammalian muscle spindles. J. Neurophysiol. 26:177-190, 1963.
Biguer B, Donaldson ML, Hein A, Jeannerod M. Neck muscle vibration modifies the representation of visual motion and direction in man. Brain 111:1405-1424, 1998.

Bless W, De GraAf B. Ocular rotation and perception of the horizontial under static tilt conditions in patients without labyrinthine function. Acta Otolaryngol. (Stockh). 111:456-462, 1991.

Burke D, Hagbarth Ke, Lofstedt L, Wallen BG. The responses of human muscle spindle endings to vibration of non-contracting muscles. J. Physiol. 261:673-693, 1976.

Dai MJ, Curthoys IS, Halmagy GM. Linear acceleration perception in the roll plane before and after unilateral vestibular neurectomy. Exp. Brain Res. 77:315-328, 1989.

de Graaf B, Bekkering H, Erasmus C, Bles W. Influence of visual, vestibular, cervical and somatosensory tilt information on ocular rotation and perception of the horizontal. J. Vest. Res. 2:15-30, 1992.

Diamond SG, Markham CH, Simpson NE, Curthoys IS. Binocular counterrolling in humans during dynamic rotation. Acta Otolaryngol. 87:490-498, 1979.

Dichgans J, Diener HC, Brandt T. Optokinetic-graviceptive interaction in different head positions. Acta Otolaryngol. 78(56):391-398, 1974.

Friedmann G. The judgement of the visual vertical and horizontal with peripheral and central vestibular lesions. Brain 93:313-328, 1970.

Goodwin FM, McCloskey DI, Matthews PB. The contribution of muscle afferents to kinaesthesia shown by vibration induced illusions of movement and by the effects of paralysing joint afferents. Brain 95:705-748, 1972.

Guerraz M, Poquin D, Luyat M, Ohlmann T. Head orientation involvement in assessment of the subjective vertical during whole body tilt. Percept. Mot. Skills 87:643-648, 1998a.

Guerraz M, Poquin D, Ohlmann T. The role of head-centric spatial reference with a static and kinetic visual disturbance. Percept. Psychophys. 60(2):287-295, 1998b.

Halmagy GM, Curthoys IS, Cremer PD, Henderson CH, Todd MH, StAPLES MJ, D'Cruz DM. The human horizontal vestibulo-ocular reflex in response to high-acceleration stimulation before and after unilateral vestibular neurectomy. Exp. Brain Res. 81(3):479-490, 1990.

Halmagy GM, Yavor RA, Colebatch JG. Tapping the head activates the vestibular system: a new use for the clinical reflex hammer. Neurology 45(10):1927-1929, 1995.

Karlberg M, Aw ST, Halmaygi GM, Black RA. Vibration-induced shift of the subjective visual horizontal. Arch. Otolaryngol. Head Neck Surg. 128(Jan):21-27, 2002.

Karlberg M, Aw ST, Black RA, Todd MJ, MacDougall HG, Halmagy GM. Vibration-induced ocular torsion and nystagmus after unilateral vestibular deafferentation. Brain 126:956-964, 2003.

Karnath H-O, Sievering D, Fetter M. The interactive contribution of neck muscle proprioception and vestibular stimulation to subjective "straight ahead" orientation in man. Exp. Brain Res. 101:140-146, 1994.

KompanejETZ S. Investigation on the counterrolling of the eyes in optimum head positions. Acta Otolaryngol. (Stockh). 12: 332.

Lackner JR, Graybiel A. Elicitation of vestibular side effects by regional vibration of the head. Aerosp. Med. 45(11):1267-1272, 1974.

LACKNER JR, LEVINE MS. Changes in apparent body orientation and sensory localization induced by vibration of postural muscles: vibratory myesthetic illusions. Aviat. Space Environ. Med. $50(4): 346-354,1979$.

Markham CH. Anatomy and physiology of otolith-controlled ocular counterrolling. Acta Otolaryngol. Suppl. 468:263-266, 1989. 
Mayoux-Benhamou MA, Revel M, Vallee C. Selective electromyography of dorsal neck muscles in humans. Exp. Brain Res. 113:353-360, 1997.

Moore KL (1980) Clinically oriented anatomy.Williams and WilkinsBaltimore/London.

Müller GE. Uber das Aubertsche Phanomenon. Z. Psychol. Physiol. Sinnesorg. 49:109-246, 1916.

Popov KE, Lekhel H, Faldon M, Bronstein AM. Visual and oculomotor responses induced by neck vibration in normal subjects and labyrinthine-defective patients. Exp. Brain Res. 128:343352, 1999.

RoLl JP, VEDEL JP. Kinaesthetic role of muscle afferents in man, studied by tendon vibration and microneurography. Exp. Brain Res. 47:177-190, 1982.

Strupp M, Arbusow V, Dieterich M, Sautier W, Brandt T. Perceptual and oculomotor effects of neck muscle vibration in vestibular neuritis. Ipsilateral somatosensory substitution of vestibular function. Brain 121:677-685, 1998.

TAYlor JL, MCCloskey DI. Illusions of head and visual target displacement induced by vibration of neck muscles. Brain 114:755-759, 1991.
Tribukait A, Bergenius J, Brantberg K. The subjective visual horizontal for different body tilts in the roll plane: characterization of normal subjects. Brain Res. Bull. 40(5/6):375-383, 1996.

WADE NJ. Visual orientation during and after lateral head, body, and trunk tilt. Percept. Psychophys 3:215-219, 1968.

WADE NJ. The effect of stimulus line variations of visual orientation with head upright and tilted. Austr. J. Psychol. 21(2):177-185, 1969.

WetZig J, von BAUMgarten RJ. Influence of neck receptor stimulation on eye rotation and on the subjective vertical: experiments on the tilt table, under water, and in weightlessness. In: Berthoz WG, Vidal PP (Eds.) The head-neck sensorimotor system. Wiley, Chichester, UK, pp 198-200, 1990.

WitkIN HA, Asch SE. Studies in space orientation. III. Perception of the upright in the absence of a visual field. J. Exp. Psychol. 38:603-614, 1948.

Young ED, Fernandez C, Goldberg JM. Responses of squirrel monkey vestibular neurons to audio-frequency sound and head vibration. Acta Otolaryngol. 84:352-360, 1977. 